INTERNATIONAL DESIGN CONFERENCE - DESIGN 2018

https://doi.org/10.21278/idc.2018.0319

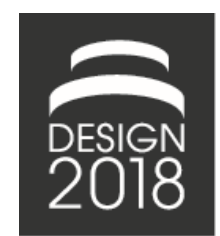

\title{
DESIGNING HUMAN-ROBOT COLLABORATIONS IN INDUSTRY 4.0: EXPLORATIVE CASE STUDIES
}

\author{
B. A. Kadir, O. Broberg and C. Souza da Conceição
}

\begin{abstract}
We are experiencing an increase in human-robot interactions and the use of collaborative robots (cobots) in industrial work systems. To make full use of cobots, it is essential to understand emerging challenges and opportunities. In this paper, we analyse three successful industrial case studies of cobots' implementation. We highlight the top three challenges and opportunities, from the empirical evidence, relate them to current available literature on the topic, and use them to identify key design factor to consider when designing industrial work system with human-robot collaborations.
\end{abstract}

Keywords: ergonomics, industry 4.0, cobot, digital manufacturing, case study

\section{Introduction}

The move towards the fourth generation industrial revolution (Industry 4.0) is promising shorter development times, increased individualised customisation, higher flexibility, and resource efficiency. It is creating the notion of a smart factory where everything is interconnected, equipped with sensors, and functions as an autonomous and self-organising system that requires minimal human intervention with the capability of adapting to human needs (Lasi et al., 2014). Industry 4.0 and the paradigm of a smart factory are bringing a wave of appertaining technological advancements that will enable the creation of smart products and services through smart processes (Preuveneers and Ilie-Zudor, 2017). This paradigm shift and digital transformation is enhancing the transparency of production processes and changing, organisational boundaries and operations of industrial companies throughout the entire supply chain, starting from the identification of the customer's need to the delivery and lifecycle management of the finished product (Stock and Seliger, 2016).

This intense focus on digitalisation and implementation of technological advancements is effecting the structure and performance of work in industrial work systems (Müller et al., 2017). Digital technologies such as Internet of Things (IoT), Cyber-Physical Systems (CPS), cloud technology, and big data are reshaping the concept of manufacturing by increasing the efficiency and effectiveness of daily collaborations, while blurring the boundaries between the physical world and the virtual space (Wang et al., 2015).

One of the more tangible technologies effecting the human-technology interactions in manufacturing is collaborative robots, also referred to as cobots. Cobots are a new generation of robots are that born free and unbounded by any type of fencing or enclosure, transcending the boundaries and workspace limitations that prevented their ancestors (standard industrial robots) from cohabitating and working side by side with their human counterparts. Equipped with sensors and being highly responsive to the detection of any unexpected force, grants them the ability to stop immediately when encountering human workers or any misplaced objects in their path. This makes them highly reliable colleagues when it comes to workplace safety, in comparison to standard industrial robots (BSI Group, 2016). The concept 
of cobots is not as novel as one would think and dates back to 1996, though it has been through an evolution since this time. First generation cobots were quite different compared to what we categorize as cobots today. While today's cobots are very similar to traditional industrial robots, (with the additional ability to work with human workers without any enclosure) first generation cobots did not have motors, were intrinsically passive in the plane of operation, and had brakes. Figure 1 shows a modern day cobot from Universal Robots.

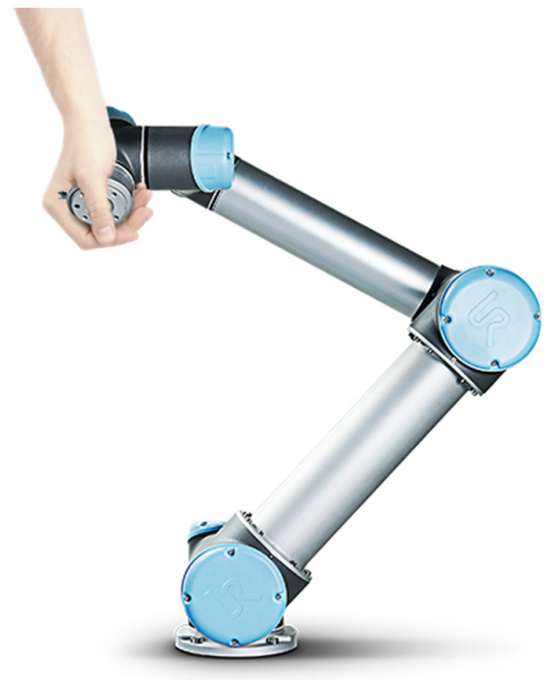

Figure 1. Universal Robot - model UR5 (Universal Robot, 2017)

Current research within the field of human-robot interactions is suggesting the need for further investigation and evaluation of challenges concerning performance, functionality, usability and environment conditions in the design and implementation of industrial work systems with cobots (Djuric et al., 2016). This paper is contributing with empirical evidence to reduce this literature gap by researching this new digital co-worker's ability to work with its human colleagues, and the changes it is evoking with its presence in industrial work system from a human factors and ergonomics perspective. We want to explore and understand the challenges and opportunities related to the implementation of cobots and this new way of working, through the eyes of the people on the shop floor as well as the decision makers that have taken part in the investment decision. Hence, the research question for this paper: What are the work and work organisation design challenges and opportunities that emerge when changing from an "old" work system to a new one where humans and collaborative robots cohabitate?

\section{Methodology}

Exploratory case studies are most appropriate when it is desired to establish an in-depth understanding of a phenomenon with research questions mainly focusing on "what?" and "how?", so as to develop relevant hypothesis and propositions to be further investigated (Yin, 2009). This coincide very well with the research question proposed in this paper and the chosen approach of conducting exploratory case studies, where the focus is to explore and identify key factors behind the acquisition of cobots, understanding the emerging opportunities and challenges and identifying key design factors in designing human-robot work systems.

\subsection{Case settings}

We have conducted three exploratory industrial case studies, investigation successful implementation of cobots and design of human-cobot teams. All three participating companies have had their cobot(s) for more than one year, and they are "typical" cases in that the cobots are performing task/tasks in work systems, which previously have consisted exclusively of manual labour performed by workers. The companies are using their cobots in different ways but the element of close human-robot interaction 
within a work system is the common theme. Table 1 shows an overview of the case companies participating in this study.

Table 1. Summary of case companies

\begin{tabular}{|l|l|l|l|l|}
\hline Company & Size & Industry & Brand and Model & Task \\
\hline A & Small $(<50)$ & Machining & Universal Robot - UR5 & Pick and place \\
\hline B & Small $(<50)$ & $\begin{array}{l}\text { Manufacture of metal structures } \\
\text { and parts of structures }\end{array}$ & Universal Robot - UR5 & Welding \\
\hline C & Large $(>250)$ & Manufacture of plastic & Universal Robot - UR5 & Assembly \\
\hline
\end{tabular}

\subsubsection{Company A}

Company A is a small machining company and manufacture of fabricated metal parts, established in 2010. It is a family owned business with several family members being a part of its fulltime staff. The company acquired their first cobot in 2011 and currently have two active ones working fulltime in their manufacturing facility, performing pick and place tasks as a part of a Computer Numerical Control (CNC) machining process. This is a repetitive task, taking around 40 seconds, which the workers manually performed prior to the implementation of the cobots. The workers' current tasks consist of feeding the cobot with unprocessed components and preparing processed components for packaging every 20-30 minutes, with each task taking around five minutes to complete. Furthermore, the worker is sporadically passing by the area to check up on the process and the cobot, and perform simple manual tasks like taking samples for quality control, or removing excess metal scrap from the CNC machine, to ensure quality and continuous production.

\subsubsection{Company $B$}

Company B, also a small family owned company with less than 50 people on its payroll, was established in the late 1970's, and is specialised in manufacturing metal structures and parts of structures. Over the last 8 years, the company has made an effort to automatize and digitalise several key processes in their manufacturing facility by e.g. implementing industrial robots and digital quality control solutions. Currently, they have two cobots working in conjunction with a human worker on a metal welding process. The cobots pick and place the metal parts and welds them together respectively. The worker prepare the unprocessed parts, starts the process and occasionally control the placement of the metal parts to ensure correct placement before welding. After the completion of a series, the worker will move the finished parts to a temporally stock. Prior to having the cobots, the worker manually performed the entire processes of picking, placing and welding the components.

\subsubsection{Company $C$}

Company C is a large manufacture of plastic established in the late 1970's with more than 350 fulltime employees working in its offices and manufacturing facilities combined. Their efforts in process automation and investment in industrial robots dates back to the mid 1980's and they currently have more than 50 industrial robots in their manufacturing facility. While these industrial robots are working in enclosed areas with restricted human contact, the single cobot in the facility is working inches away from its human colleagues, and providing assistance in the assembly of one of the companies many specialised products. The human worker will place the product on the workstation, insert a small part of a long rubber band into the product and signal the cobot by the push of a button to take over and complete the insertion of the rubber band. While the cobot is performing this task, the human worker will prepare another product, and insert rubber bumpers in the products already processed by the cobot. Before the company acquired the cobot, the worker was manually performing all of the tasks.

\subsection{Data collection}

We collected qualitative data through semi-structured interviews with decision makers (production manager, CEOs) that are responsible are the acquisition and implementation of the cobots, workers that work side-by-side with the cobots, and through observations and demonstrations of the work system 
and human-robot collaborations in action. Additionally, we interviewed a consultant within the field of cobots, whom has assisted several companies with the implementation of cobots. All interviews were carried out in face-to-face settings with durations between 20-60 minutes and audio-recorded and transcribed in Danish. Using a participant observation type (Krause and Denzin, 1989), observations and demonstration of the work systems were video-recorded with consent from the authoritative managers at the companies and relevant field notes were taken according to (Spradley, 1980). Table 2 shows an overview of the interview participants categorised by their position and role.

Table 2. Summary of collected qualitative data

\begin{tabular}{|c|c|c|c|}
\hline Company & Data collection method & Description & Referred as \\
\hline \multirow[t]{4}{*}{ A } & \multirow[t]{3}{*}{ Interview } & Worker & A1 \\
\hline & & Worker & A2 \\
\hline & & CEO & A3 \\
\hline & Observation & $\begin{array}{l}\text { Workspace and human-robot collaboration in } \\
\text { action }\end{array}$ & - \\
\hline \multirow[t]{3}{*}{ B } & \multirow[t]{2}{*}{ Interview } & Worker & $\mathrm{B} 1$ \\
\hline & & $\mathrm{CEO}$ & B2 \\
\hline & Demonstration & Demonstration of human-robot collaboration & - \\
\hline \multirow[t]{3}{*}{$\mathrm{C}$} & \multirow[t]{2}{*}{ Interview } & worker & $\mathrm{C} 1$ \\
\hline & & Production manager & $\mathrm{C} 2$ \\
\hline & Demonstration & Demonstration of human-robot collaboration & - \\
\hline Consultancy & Interview & Interview with consultant & Consultant \\
\hline
\end{tabular}

\subsection{Data analysis}

We have used a general inductive approach (Thomas, 2006) to develop relevant theories and present findings based on summaries of the data collected through interviews and observations and have established relevant links between the findings and the proposed research. The first author has coded and constantly compared the collected data accordingly with the emergence of new categories throughout the process and made use of template analysis (Brooks et al., 2015) to analyse the data according to the themes related to the research question. While the general inductive approach is used as a general approach for the overall analysis, the data generated from the interviews was compared with a purposeful approach to the comparative method (Boeije, 2002), which is an essential part of the grounded theory approach. We have used the data collected from the observations and demonstrations to verify information and statements made in the interviews and to search for and uncover additional information not mentioned or left out in the interviews.

\section{Results}

The analysis of the qualitative data focused on identifying work and work organisation design challenges and opportunities that emerged when the companies introduced cobots into their work systems. In the following two sub-sections, we highlight and elaborate on the most frequently mentioned challenges and opportunities.

\subsection{Challenges}

\subsubsection{Cobots are not reflective}

While cobots are sensitive to contact and safe to work with, they still lack the flexibility of their human colleagues and the ability to improvise, adapt and overcome. They can be programmed to perform detailed tasks with precision, but their lack of ability to detect abnormalities such as; variation in raw material or correct placement of components is making them less flexible compared to the human workers. This disadvantage is leaving the workers with the responsibility of ensuring the quality of the work performed by the cobots. All of the interviewed workers, as well as the decision makers from 
company $\mathrm{A}$ and $\mathrm{C}$ mentioned this challenge. The production manager at company $\mathrm{C}$ explained how this challenge could eventually lead to frustration amongst the workers leaving them to distrust the cobots. He viewed this as the decisive different between workers and cobots, which his company was making an effort to solve.

C2: "We humans are very flexible... If the rubber band received from the supplier is a bit too long, we will compensate as a human, and push or pull it a little bit. The robot will not do that" ... people will get frustrated and start doubting the technology thinking that it is not working".

One of the workers at company A added the following about the cobot's ability to detect abnormalities. A1: "I'll check up on the machine (CNC machine) continuously. If something breaks inside the machine, the product measurements will be completely off. But the machine has no alarms and the robot will not be able to detect if anything is wrong and just continue producing bad products."

\subsubsection{Cobot implementation does not make any sense}

Cobots might be more affordable compared to a standard industrial robot, but they are still coming with a price tag that is considerable for most small and medium-sized enterprises (SMEs). When a company is acquiring a cobot, they usually have a specific task in mind, which they want the cobot to occupy, but since the technology is relatively new and untested, the implementation and achieved results might not always turn out as expected. In the situation where it is not possible to use the cobot as intended, the company has to make a decision that can justify their investment in the technology by utilising the cobot in other human-cobot collaboration tasks in the company. The workers and decision makers at both company $\mathrm{B}$ and $\mathrm{C}$ mentioned this challenge.

The production manager from company $\mathrm{C}$ mentioned that the specific task the cobot was intended for had been put on stand-by, because the customer had decided to delay its order at the last moment. In the meantime, they are using the cobot for a similar task on another product, though with a much lower production volume and frequency leaving the cobot underutilised and often unused. Creating other tasks for the robot seem to be challenging. Company $\mathrm{C}$ is currently collaborating with a university student on his bachelor thesis focusing on finding new tasks for the cobot.

C2: "We want to use the cobot as intended, so it is important to create tasks with the right flow, so it becomes a collaboration and not the cobot doing all of the tasks while the worker is sitting is simply observing on the side."

Company B had experienced similar difficulties. About a year prior to having the current setup of using two collaborative robots for welding metal parts, in their initial attempt, they decided to sell their collaborative robot after having it for only one year, due to inefficient utilisation that could justify the investment and continuation with the technology.

B2: "When we have had it (the collaborative robot) for about a year, we realised that it did not make any sense. We took it down and sold it. It did not make any sense".

Apparently, this is a common challenges faced by many companies that have tried their luck with implementing cobots. The consultant mentioned that there are many collaborative robots stored in basements, collecting dust, because the companies did not manage to utilise the technology as intended, leading to impractical and non-functioning human-robot collaboration.

\subsubsection{Cobots can hit workers}

Sharing a workspace with a non-human entity that is able to roam freely in a fixed pattern without any enclosure is a relatively new type of setup. This might seem a bit freighting and evoke worries for some workers, since they are not familiar with this way of working. It will require an adjustment of movement patterns, behaviour and way of thinking before they can feel comfortable working alongside the cobots. Both workers from company A and the worker from company $\mathrm{C}$ mentioned their initial worries about being hit by the cobot, even when they were aware of its safety features and its ability to stop instantly if they came in contact with it.

A1: "At the beginning, before I was familiar with the cobot and its movement pattern, I was worried about the cobot hitting me".

Though the workers had initial worries regarding their safety, they all expressed that it quickly passed after having worked with the cobot for a while. The decision makers were also very sure about their 
workers safety around the cobots. The production manager at company $\mathrm{C}$ explained that the work cell they integrated their cobot in, was obligated to have a CE (Conformité Européenne) marking. It should be mentioned that the $\mathrm{CE}$ marking is an internal certification and does not require involvement from a third party certification body (European Commission, 2018).

C2: "When you build a work cell like ours, you are obligated to get a CE marking, and that CE marking contains a risk evaluation where we look at every single part of the work process and assess the risk of getting a finger squeezed or what else might happen along the process."

The safety features of the cobots seem to be comforting, but the workers still have to overcome their initial worries about being hit. To comfort his workers and to ensure them of the cobots safety features, the production manager at company $\mathrm{C}$ had given a demonstration by putting his own hand in an uncompromising position where it easily would have been squeezed and injured if the cobot did not possess its safety features. All of the workers, except for $\mathrm{C} 1$ had tried getting hit by the cobot and experienced it stopping automatically and immediately. The experience was in all accounts described as painless and insignificant.

\subsection{Opportunities}

\subsubsection{Cobots enable job enrichment}

The cobots are taking the repetitive and trivial manual work away from the workers and leaving them with more time to spend on other and new potentially value creating activities. The decision makers as well as workers all consider this as one of the greatest benefits that derive from the implementation of the cobots. Due to their flexibility and quick learning ability, the worker are able to occupy new functions and take on new roles and responsibilities, which they previously did not have the opportunity and time for. Workers at the case companies had added the following as a part of their job; preparing products for storage, cleaning and servicing machines, programming cobots and other machines, designing new collaborative work with cobots, management of small internal projects, quality control, production planning, making technical drawings, and performing other manual tasks in the production. It should be noted, that none of the workers have officially had their job descriptions updated to encompass these new roles and responsibilities.

A3: "We are being forced to lower our prices, since everyone was outsourcing to Eastern Europe... in order to retain our business, we had to free up some of our workers time so they could assist with other tasks. So, we let the robots take over the repetitive parts of the job"

The consultant we interviewed highlighted that the companies he assists usually desire a solution where the cobot might be taking over around $60-80 \%$ of a given task, which in most cases are the repetitive and trivial parts of the job, leaving the workers to perform the rest of the remaining and more mentally demanding parts of the job. Seen from the company's perspective, this is a great opportunity to utilise their work force more efficiently and assign new tasks and responsibilities to the workers. Company B and $\mathrm{C}$ have used this opportunity to further educate and develop their workers skills and competences by having them participate in relevant courses. The workers all seemed to be very satisfied with this arrangement, as they get to try their hands on new tasks, gain more responsibilities and develop new skills in the process.

A1: "... Before when we didn't have the collaborative robot, I was switching the parts manually one by one. That can be very trivial and boring over time. Therefore, it is nice that we now have a robot to take care of that part of the job, so I get the opportunity to try other tasks... I do other value creating tasks in the production."

\subsubsection{Cobots improve workflow}

The implementation of cobots have resulted in improved workflow and continuous production. Humanrobot teams are much more efficient compared to teams consisting of humans only, due to the fluency of the collaborative effort, where the cobot is performing the repetitive and physically demanding tasks, while the worker is taking care of the more flexible and high variation parts of the tasks. This emerged opportunity was mentioned by the workers and decision makers at company A, as well as the decision 
makers at company $\mathrm{B}$ and $\mathrm{C}$. By implementing the cobot, company $\mathrm{C}$ has been able to reduce the assembly time by $50 \%$ for that specific work cell, going from two minutes to one.

This continuity has also had its positive effect at company A, which has been able to add evening and night shifts consisting of two workers and two cobots. Previously, this would not have been possible, since the company could not afford having several more employees working these shifts, to produce an output equal to the current setup with the cobots. Company $\mathrm{C}$ is also seeing the benefits of this improved workflow. However, they attribute this to their overall effort and investment in cobots as well as other technologies implemented throughout their facility.

C2: "When considering how we were working in the past, and how we are working now, the working environment is much better". The quality is improved and what we are much more punctual when delivering to our customers. The entire flow in the company has improved"

\subsubsection{Cobots take away repetitive tasks}

The cobots are taking away the most repetitive tasks from the workers and it is having a considerable effect on the physical work conditions and the workers wellbeing. The workers are getting rid of physically demanding tasks and the decision makers are pleased to have healthy and well-functioning workers that can perform optimally for many years to come. All workers and decision makers except A2 highlighted this opportunity.C1: "It is great having it take away this part of the tasks. When I do it manually, I experience wrist pain... The cobot is getting rid of some parts of the tasks that are physically demanding, monotonous and repetitive... We have had a quit many workers getting injured or worn out because they were doing repetitive work"

A1: "Previously, I was manually inserting the components one at a time and had to bend over and into the machine 600 times pr. Day... So, seen from a work environment and health perspective, it is removing the parts of the job, which were causing me a lot of back pain"

Even though the all decision makers made it clear that economics was the driving factor behind the investment, they also expressed great satisfaction with the cobots' ability to improve physical work conditions and their contribution to a better and healthier work environment.

\subsection{Summary of results}

The results indicates that the case companies had been able to setup and install the cobots relatively quickly and easily. The workers feel safe around them and their arrival has caused a reduction in repetitive and trivial work, which has led to improved physical work conditions and given the workers room to do other potentially value creating tasks. While the installation of these cobots might be easy, finding the right tasks and making efficient use of their capabilities seem to be challenging. The lack of surrounding enclosure restricts them from operating at speeds matching standard industrial robots, and their lack of ability to detect abnormalities makes them less flexible compared to their human colleagues.

\section{Discussion}

The current literature available on the topic of human-robot interaction and collaborative robots in industrial work systems is indicating several implementation, work, and work organisation design challenges and opportunities. However, because the technology is relatively new and in its infant stage, there is still a need for more empirical evidence that can highlight and address the appertaining challenges and opportunities. This paper contributes to reducing this gap with empirical evidence. Empirical evidence based literature dealing with this specific topic is limited, hence the relevancy of this paper and the obtained results. What we have identified as the most frequently mentioned challenges

opportunities in this paper are similar to the findings of Djuric et al. (2016) who gives an overview of some of the challenges of cobots, based on a literature review on the topic. However, there is no direct mentioning of the cobots' ability to enable job enrichment by allowing the workers to take on new tasks, develop new skills and professional competences, which we have identified as one of the top three emerging opportunity.

The most frequently mentioned challenge was the cobots inability to reflect and handle component- and process variability, which is a similarly well-known challenge associated with standard industrial robots. 
However, this can also be viewed as one of the benefits of cobots in comparison to industrial robots, since the cobots can work side by side with a workers that can perform the complex tasks that require sensory inputs and creativity (Matthias et al., 2011). To have standard industrial robots accommodate this challenge will require implementation of advanced sensors and vision systems, which can be very demanding and expensive (Krüger et al., 2009).

If a company is not able to justify their investment, it will no longer make any sense to keep the cobot, as it was the case in company B's first attempt at implementing cobots. In order to utilise the cobots to their full potential and realise the advantages of human-robot collaboration, it is important to have designed well-defined tasks and work organisation, which requires a vast understanding of the work system as well as the capabilities of the technology (Grahn and Langbeck, 2005). This correlates with the challenge of the cobots not being able to reflect. When the cobots are efficiently utilised, they will allow the workers to spend time on working on other tasks within their companies. While it was clear that the decision makers and the workers had a pretty god idea of what these new tasks were, none of them had clearly defined or officially documented them.

Work safety and risk management is an essential part of designing human-robot collaborations and must follow regulated safety standards such as (BSI Group, 2016). Unclear understanding of the technology might result in insufficient planning and considerations for physical work and work safety. It is understandable that the workers from the case companies had initial worries about being hit by the cobots while working in close proximity to the cobots, considering that most injuries in modern manufacturing have historically happened due to unintentional contact between robot and workers (Marvel et al., 2015). Cobots are being promoted as safe to work with, but that does not mean that they can be implemented into a work system without any safety considerations. The Institute for Occupational Safety and Health of German Social Accidents Insurance (BG/BGIA) has developed a guideline and recommendations that can be used for the assessment of risk in the design of workplaces with collaborative robots (Bgia, 2009). Since the challenges and opportunities seem to be highly correlated, it can be argued that the all of the challenges must be addressed in order to achieve the full potential benefits of the technology.

\subsection{Implication for practitioners}

Designing a successful human-robot collaboration in industrial work systems starts prior to the decision of investment has been finalised. It is important to have realistic expectations of the cobot's ability to perform and manage the tasks you wish to assign upon it and that you are in agreement with the quality the technology is able to deliver (Robotiq, 2017). Successful implementation requires the company to have an in-depth understanding of the existing work system and have created a plan for division of work between workers and cobots. Based on the challenges and opportunities highlighted in this paper, we have identified five key design factors for practitioners to consider when designing work systems with cobots. In any case, it is important to follow and adhere to standards such as (BSI Group, 2016) and conduct the necessary risk assessments and work evaluations when implementing cobots and designing new or re-designing current work systems, since they provide a good starting point and a solid foundation for implementation.

\subsubsection{Understanding existing processes}

Before investing in cobots, it is important that the company starts by visualising the processes of the existing work system in order to gain the necessary understanding of material- and workflow and to identify co-dependencies and co-relations within the work system. Having this understanding will lead to better decisions regarding role assignment, work organisation, and work division between cobots and workers.

\subsubsection{Clear task division between humans and cobots}

When you have a comprehensive understanding of the work system, it should be easier to create a clear task division between cobot and workers. This division of tasks should be based on the capabilities and skills of the cobots and workers, leaving the repetitive and monotonous work to the cobots, and flexible, complex and creative tasks to the workers. In order to create an efficient and effective work system as 
well as reducing frustration and concerns from the workers, it is essential to clearly define, and document the new tasks, roles and responsibilities of the workers when designing the work system.

\subsubsection{Visualise movement paths and workspace}

Using visual cues and guides such as colour tape markings to highlight the cobot's movement paths and the division of the workspace between the workers and the cobots could create more awareness and reducing coalitions between the workers and the cobots. This could have an impact on the workers perception of safety and making them more confident and secure while working in close proximity to the cobots. Furthermore, visual guides can increase the efficiency of the manufacturing work environment and create flexibility by providing every worker with the same visual information.

\subsubsection{Developing standard operating procedures and train workers}

Work efficiency, output consistency and the learning rate of the people working within a work system can be enhanced with standard operating procedures (SOP). We do not suggest SMEs to create detailed SOPs consisting of several pages but rather an updatable one-page job instruction that explains the overall process steps. As the workers gain experience of working with the cobots, work- and process improvement opportunities will emerge. However, without standardised procedures it will be difficult to implement any effective and sustainable improvements.

\subsubsection{Systematic quality control}

The cobots' inability to reflect and detect abnormalities might result in poor work quality. Therefore developing a systematic quality control procedure could have a positive impact on the quality of the work performed by the cobots. Systematic quality control will reduce product scrap and defects, machine stops, lost equipment time and worker frustration. This could be a systematic visual control of the cobots and the process, following a simple check-sheet on a fixed schedule.

\subsection{Limitations and further research}

The limitation of this paper is the number of case companies and interviews conducted, and the limited data on failed implementations of cobots. Further research could include using the data from the paper combined with more qualitative data from new industrial case companies, collected in the same manner to develop a framework for designing industrial work systems with human-robot collaborations.

\section{Conclusion}

In this paper, we conducted three explorative cases studies to examine the work and work organisation design challenges and opportunities. Since the identified challenges and opportunities are highly correlated, we argue that the all of the challenges need to be addressed before it is possible to achieve the full potential benefits of the technology. Additionally we have compared our findings with the literature available on the topic and used the outcome to identify five key design factors that should be considered when designing human-robot collaborations in industrial work systems.

\section{References}

Bgia (2009), BG/BGIA risk assessment recommendations according to machinery directive: Design of workplaces with collaborative robots. [online] Available at: www.dguv.de/bgia (accessed 6.12.2017).

Boeije, H. (2002), "A purposeful approach to the constant comparative method in the analysis of qualitative interviews", Quality and Quantity, Vol. 36 No. 4, pp. 391-409.

Brooks, J., McCluskey, S., Turley, E. and King, N. (2015), "The Utility of Template Analysis in Qualitative Psychology Research", Qualitative Research in Psychology, Vol. 12 No. 2, pp. 202-222.

BSI Group (2016), Robots and robotic devices - Collaborative robots (ISO / TS 15066 : 2016), BSI Standards Publication.

Djuric, A.M., Urbanic, R.J. and Rickli, J.L. (2016), “A Framework for Collaborative Robot (CoBot) Integration in Advanced Manufacturing Systems”, SAE International Journal of Materials and Manufacturing, Vol. 9 No. 2, pp. 2016-01-0337. 
European Commission (2018), Manufacturers - European Commission, CE Marking. [online] Available at: https://ec.europa.eu/growth/single-market/ce-marking/manufacturers_en (accessed 23.2.2018).

Grahn, S. and Langbeck, B. (2005), Benefits of Collaborative Robots in Assembly - an Evaluation Scheme. [online] Available at: http://www.ipr.mdh.se/pdf_publications/3806.pdf (accessed 6.12.2017).

Krause, D. and Denzin, N.K. (1989), "The Research Act: A Theoretical Introduction to Sociological Methods", Teaching Sociology, Vol. 17 No. 4, p. 500.

Krüger, J., Lien, T.K. and Verl, A. (2009), "Cooperation of human and machines in assembly lines", CIRP Annals - Manufacturing Technology, Vol. 58 No. 2, pp. 628-646.

Lasi, H., Fettke, P., Kemper, H.-G., Feld, T. and Hoffmann, M. (2014), “Industry 4.0”, Business \& Information Systems Engineering, Vol. 6 No. 4, pp. 239-242.

Marvel, J.A., Falco, J. and Marstio, I. (2015), "Characterizing Task-Based Human-Robot Collaboration Safety in Manufacturing”, IEEE Transactions on Systems, Man, and Cybernetics: Systems, Vol. 45 No. 2, pp. $260-275$.

Matthias, B., Kock, S., Jerregard, H., Kallman, M. and Lundberg, I. (2011), "Safety of collaborative industrial robots: Certification possibilities for a collaborative assembly robot concept", 2011 IEEE International Symposium on Assembly and Manufacturing (ISAM), IEEE, pp. 1-6.

Müller, S.L., Shehadeh, M.A., Schröder, S., Richert, A. and Jeschke, S. (2017), “An overview of work analysis instruments for hybrid production workplaces", AI \& SOCIETY. https://doi.org/10.1007/s00146-017-0757-9

Preuveneers, D. and Ilie-Zudor, E. (2017), "The intelligent industry of the future: A survey on emerging trends, research challenges and opportunities in Industry 4.0", Journal of Ambient Intelligence and Smart Environments, Vol. 9 No. 3, pp. 287-298.

Robotiq (2017), Collaborative Robots Buyer's Guide, 7th ed. [online] Robotiq. Available at: https://cdn2.hubspot.net/hubfs/13401/COBOT EBOOK FINAL6.pdf (accessed 6.12.2017).

Spradley, J.P. (1980), "Summary for Policymakers", Intergovernmental Panel on Climate Change, Climate Change 2013 - The Physical Science Basis, Cambridge University Press, Cambridge, pp. 1-30.

Stock, T. and Seliger, G. (2016), "Opportunities of Sustainable Manufacturing in Industry 4.0", Procedia CIRP, Vol. 40, pp. 536-541.

Thomas, D.R. (2006), “A General Inductive Approach for Analyzing Qualitative Evaluation Data”, American Journal of Evaluation, Vol. 27 No. 2, pp. 237-246.

Universal Robot (2017), Universal Robots UR5. Universal Robots. [online] Available at: https://www.universalrobots.com/products/ur5-robot

Wang, L., Törngren, M. and Onori, M. (2015), "Current status and advancement of cyber-physical systems in manufacturing”, Journal of Manufacturing Systems, Vol. 37, pp. 517-527.

Yin, R.K. (2009), Case Study Research: Design and Methods, Applied Social Research Method Series, 4th ed., SAGE Publications Inc, Thousand Oaks, California.

Bzhwen A Kadir, PhD student

Technical University of Denmark, Management Engineering

Praestekaervej 13, 2. th, 2700 Broenshoej, Denmark

Email: bkad@dtu.dk 